\title{
Tópicos contrastivos com sintagmas cardinais complexos
}

\author{
Contrastive topics with complex cardinal phrases
}

\author{
Marcelo Barra Ferreira \\ Universidade de São Paulo - USP
}

Resumo

Este artigo discute uma diferença de significado entre as expressões aparentemente sinônimas no mínimo $n$ (máximo $n$ ) e mais de $n-1$ (menos de n+1). Proponho uma explicação semântico-pragmática baseada em princípios gerais que regulam o uso de perguntas e respostas durante o fluxo de uma conversa e na proposta de Buring (1997, 2003) de que expressões linguísticas possuem, além de um valor semântico ordinário, um valor de foco e um valor de tópico. A ideia central é que no mínimo e no máximo trazem do léxico um acento de tópico, o que determina um valor de tópico não trivial e faz com que seu uso esteja sujeito a condições que o uso de mais de e menos de não estão.

Palavras-Chave

Cardinais complexos, Tópico contrastivo, Perguntas.

\section{Abstract}

This article discusses a difference in interpretation between the apparently synonymous expressions at least $n$ (at most $n$ ) and more than $n$-1(fewer than $n+1)$. I propose a semantic-pragmatic explanation, based on general principles that regulate the use of questions and answers during a conversation and on ideas proposed by Buring $(1997,2003)$, according to whom linguistic expressions 
are associated with an ordinary semantic value, a focus value and a topic value. The central idea is that at least / at most are lexically marked with a topic accent, which affects their topic value in a non trivial way and makes their use subject to conditions that do not apply to more than / fewer than.

\section{Keywords}

Complex cardinals, Contrastive topic, Questions. 


\section{Introdução}

intagmas nominais cardinais podem ser formados por um numeral - seguido de uma expressão nominal como três carros em (1) abaixo, ou podem vir acompanhados de expressōes como no minimo, mais de, no máximo, menos de, como nos exemplos em (2) e (3):

(1) Jonas tem três carros.

(2) a. Jonas tem no mínimo três carros.

b. Jonas tem mais de dois carros.

(3) a. Jonas tem no máximo três carros.

b. Jonas tem menos de quatro carros.

Enquanto (1) parece veicular informação sobre o valor exato da cardinalidade do conjunto de carros que o Jonas tem, os exemplos em (2) e (3) identificam apenas parcialmente este valor, impondo ou um limite inferior para esse valor, como em (2), ou um limite superior, como em (3). ${ }^{1}$ À primeira vista, portanto, ambas as sentenças em (2) parecem equivalentes do ponto de vista de seus conteúdos proposicionais, podendo estes ser representados formalmente como em (4):

(4) $\exists X:|X| \geq 3 \& \operatorname{carros}^{\prime}(\mathrm{X}) \& \operatorname{ter}^{\prime}(\mathrm{j}, \mathrm{X})$

O mesmo se pode dizer das sentenças em (3):

(5) $\exists X:|X| \geq 3 \& \operatorname{carros}^{\prime}(X) \& \operatorname{ter}^{\prime}(\mathrm{j}, \mathrm{X})$

O propósito deste artigo é chamar a atenção para o fato de que a despeito da aparente semelhança entre os significados das expressóes no minimo ne mais de $n-1$, por um lado, e no máximo n e menos de $n+1$, por outro, há diferenças interessantes entre elas e propor uma explicação semântico-pragmática baseada em princípios gerais que regulam o uso de perguntas e respostas durante o fluxo de uma conversa. 
O artigo está organizado da seguinte forma: na segunda seção, apresento as assimetrias mencionadas acima e discuto se seu caráter é ou não estritamente semântico; na terceira seção, forneço o background relevante para a compreensão de minha proposta, com relevo para a distribuição de acentos de foco e tópico contrastivo; na quarta seção, apresento minha proposta e, na quinta seção, termino com uma conclusão sucinta e alguns pontos deixados em aberto.

\section{Assimetrias entre os Modificadores Cardinais}

A introdução da seção anterior enfatizou uma assimetria entre sintagmas cardinais simples de um lado e sintagmas cardinais complexos de outro. Vamos passar agora a um tipo diferente de exemplo, que aponta para uma assimetria entre os próprios cardinais complexos. Essa diferença está particularmente bem ilustrada nos diálogos abaixo, tomados de Nilsen (2007):

(6) A: Dá pra ver que seu menino é filho único.

B: Não! Eu tenho mais de um filho.

(7) A: Dá pra ver seu menino é filho único.

B: \# Não! Eu tenho no mínimo dois filhos.

Enquanto a resposta de B em (6) parece perfeitamente adequada, o mesmo não se pode dizer de sua resposta em (7). Intuitivamente, a razão para essa assimetria é que em (7) B parece sugerir não saber quantos filhos tem. Dito de outra forma, a resposta sugere que até que ponto B sabe, é possível que ele tenha (exatamente) dois filhos, mas é também possível que ele tenha mais de dois. Tal efeito não aparece em (6), no qual a expressão mais de um foi usada no lugar de no mínimo 2. Assim, ainda que ambas as expressōes possam ser usadas para impor um limite inferior na cardinalidade de um determinado conjunto (nesse caso, o conjunto dos filhos de B), elas o fazem de maneira distinta, sendo que apenas o uso de no minimo legitima o que podemos chamar de uma inferência de ignorância.

(8) a. Eu tenho mais de um filho.

Inferência de Ignorância: nenhuma 
b. Eu tenho no mínimo dois filhos.

Inferência de Ignorância: É possível que eu tenha (exatamente) dois filhos, mas é possível também que eu tenha mais de dois.

Fatos similares ocorrem se substituírmos mais de e pelo menos por suas contrapartes menos de e no máximo, as quais estabelecem um limite superior para a cardinalidade de um conjunto:

(9) A: Fiquei sabendo que você tem cinco filhos!

B: Não! Eu tenho menos de cinco (filhos).

(10) A: Fiquei sabendo que você tem cinco filhos!

B: \# Não! Eu tenho no máximo quatro (filhos).

(11) a. Eu tenho menos de cinco filhos.

Inferência de ignorância: nenhuma

b. Eu tenho no máximo quatro filhos.

Inferência de ignorância: É possível que eu tenha (exatamente) quatro filhos, mas é possível também que eu tenha menos de quatro.

A divisão relevante parece ser então entre mais de / menos de por um lado e no mínimo / no máximo de outro.

É importante enfatizar que, nos diálogos acima, o que era diretamente relevante para a conversa no momento da resposta de $\mathrm{B}$ não era o número exato de filhos de B, mas, sim, se B tinha ou não um certo número de filhos. Dessa forma, não se pode dizer que em (7) ou (10) B estejam fornecendo apenas informação parcial para o tópico da conversa. E se, de fato, B não estiver retendo informação relevante, a inferência de ignorância que detectamos acima e que associamos ao uso de no mínimo / no máximo não pode estar diretamente ligada às máximas de quantidade de Grice. Obviamente, tal explicação estaria disponível se números exatos fossem o tópico em questão, como no diálogo abaixo: 
(12) A: Quantos filhos você tem?

B: \# Eu tenho no mínimo três (filhos).

B: \# Eu tenho no máximo cinco (filhos).

Mas note que nessas circunstâncias também o uso de mais de / menos de soaria estranho, sugerindo ignorância por parte do falante: ${ }^{2}$

(13) A: Quantos filhos você tem?

B: \# Eu tenho mais de três (filhos).

B: \# Eu tenho menos de cinco (filhos).

É importante, pois, ter em mente que, mesmo quando números exatos não estão em questão, o uso de no mínimo / no máximo dá margem à inferências de ignorância do tipo que acabamos de discutir.

A questão que surge neste instante é se essas inferências são de natureza puramente semântica, consequência do sentido literal das expressões no mínimo I no máximo ou se sua explicação requer recurso a princípios de ordem pragmática. Minha posição é a de que os efeitos acima não podem ser explicados em termos puramente semânticos, já que esses desaparecem em certas circunstâncias. Considere, por exemplo, a sentença abaixo, em que pelo menos aparece no interior da expressão que restringe o escopo do determinante universal:

(14) Todo aluno que respondeu no mínimo duas questôes corretamente foi aprovado.

Note que a generalização acima abarca todos os alunos que responderam duas questões, todos os que responderam três questóes, todos os que responderam quatro questôes, etc. Ela não exclui aqueles alunos que acertaram duas ou mais questões, mas cujo número exato de acertos é conhecido pelo falante. Nem tampouco (14) exprime incerteza por parte do falante em relação ao número mínimo de acertos que garantiu a aprovação. Com isso quero mostrar que o efeito de ignorância introduzido por no minimo nos enunciados que vínhamos considerando até aqui não está presente nem no nível do restritor do operador universal todo, nem no nível da oração matriz em (14). Convém notar a esse respeito que a substituição de pelo menos dois por mais de um em (14) não resulta em nenhuma diferença, conforme pode ser visto comparando (14) com 
(15) abaixo:

(15) Todo aluno que respondeu mais de uma questão corretamente foi aprovado.

O ponto relevante aqui é que essa similaridade seria inesperada se a inferência de ignorância fosse parte do sentido literal da expressão no mínimo. ${ }^{3}$

Tomarei tal fato como evidência de que as inferências de ignorância que estamos estudando não são em sentido estrito um fenômeno lógico-semântico e apresentarei uma análise que pode ser considerada parcialmente semântica e parcialmente pragmática, conforme ficará claro já a partir da próxima seção.

\section{Sobre perguntas e respostas}

Considere os dois pares pergunta-resposta abaixo:

(16) A: Quem o Jonas apresentou pra Maria?

B: O Jonas apresentou o PEDRO pra Maria.

(17) A: Pra quem o Jonas apresentou o Pedro.

B: O Jonas apresentou o Pedro pra MARIA.

Por um lado, as respostas de B em (16) e (17) são semelhantes, ambas sendo verdadeiras se, e somente se, Jonas tiver apresentado Pedro pra Maria. Podemos dizer que elas compartilham o mesmo conteúdo proposicional. Por outro lado, elas se distinguem pelo fato de que em (16), Pedro recebe um acento especial, ao passo que em (17) é Maria que recebe esse mesmo acento (indicado pelas letras maiúsculas acima), chamado de acento de foco. Essa diferença fonética tem repercussão nas condições de uso das respostas. Isso fica claro, ao notarmos que se respondêssemos a pergunta de A em (16) com a resposta de B em (17), o resultado seria, claramente, inadequado. O mesmo ocorreria se respondêssemos a pergunta em (17) com a resposta em (16). Diz-se nesse caso que essas respostas possuem valores de foco diferentes, já que, na primeira, Pedro é o elemento focalizado, enquanto que na segunda, esse elemento é Maria.

Adotando o formalismo de Rooth (1985, 1996), cada expressão linguística a é associada a dois valores semânticos: o valor ordinário $\left([[\alpha]]^{\circ}\right)$ e o 
valor de foco $\left([[\alpha]]^{\mathrm{f}}\right)$. No caso de sentenças, o valor ordinário é uma proposição, ao passo que o valor de foco é um conjunto de proposiçóes, obtidas pela substituição do elemento focalizado por alternativas do mesmo tipo semântico. Para os exemplos acima, temos o seguinte: ${ }^{4}$

(18) $\left[\left[\text { O Jonas apresentou o }[\text { Pedro }]_{\mathrm{F}} \text { pra Maria }\right]\right]^{\circ}=$ $\left[\left[\text { O Jonas apresentou o Pedro pra }[\text { Maria }]_{\mathrm{F}}\right]\right]^{\circ}=$ O Jonas apresentou o Pedro pra Maria.

(19) $\left[\left[\text { O Jonas apresentou o }[\text { Pedro }]_{\mathrm{F}} \text { pra Maria }\right]\right]^{\mathrm{f}}=$ $\{$ Jonas apresentou o Pedro pra Maria, Jonas apresentou o Paulo pra Maria, Jonas apresentou o Lucas pra Maria, ... \}

(20) $\left[\left[\text { O Jonas apresentou o Pedro pra }[\text { Maria }]_{F}\right]\right]^{\mathrm{f}}=$ $\{$ Jonas apresentou o Pedro pra Maria, Jonas apresentou o Pedro pra Paula, Jonas apresentou o Pedro pra Marta, ... \}

Rooth assume ainda, seguindo Hamblin (1973), que o valor semântico ordinário de uma pergunta é o conjunto de suas possíveis respostas, o que para nossos propósitos pode ser entendido como o conjunto das proposiçóes que se obtém ao substituir a palavra interrogativa por um nome, conforme exemplificado abaixo:

(21) [[ Quem o Jonas apresentou pra Maria? ] ] ${ }^{\circ}=$

$\{$ Jonas apresentou o Pedro pra Maria,

Jonas apresentou o Paulo pra Maria, Jonas apresentou o Lucas pra Maria, ... \}

(22) $\left[[\text { Pra quem o Jonas apresentou o Pedro? ] }]^{\mathrm{f}}=\right.$ $\{$ Jonas apresentou o Pedro pra Maria, Jonas apresentou o Pedro pra Paula, Jonas apresentou o Pedro pra Marta, ... $\}$ 
Note que o valor ordinário da pergunta em (21) é igual ao valor de foco da sentença em (19) e que o valor ordinário da pergunta em (22) é igual ao valor ordinário da sentença em (20). Essa equivalência está na base da seguinte condição de uso envolvendo pares pergunta-resposta (cf. ROOTH, 1996):

\section{Congruência Pergunta-Resposta}

Dadas uma pergunta $Q$ e uma resposta $R,[[\mathrm{Q}]]^{\circ}$ deve ser igual a $[[\mathrm{R}]]^{\mathrm{f}}$

É em função dessa condição que as respostas de B nos nossos exemplos iniciais em (16) e (17) não são intercambiáveis.

Esse aparato teórico se estende a perguntas sim-não, como em (24) abaixo, e as respostas possíveis nesse caso se reduzem a duas: ${ }^{5}$

(24) $\left[[\text { O Jonas chegou? ] }]^{\circ}=\{\mathrm{O}\right.$ Jonas chegou, $\mathrm{O}$ Jonas não chegou $\}$

(25) A: O Jonas chegou?

B: CHEGOU.

B': NÂO (chegou).

Sem entrar em detalhes sobre a estrutura sintática das respostas acima (cf. LAKA, 1990 e MARTINS, 1994, para discussão e referências), vamos assumir que o foco nesses casos recai sobre um operador assertivo, correspondendo à palavra não nas respostas negativas e a um morfema abstrato sem realização fonética nas respostas afirmativas (o acento de foco nesse caso apoiando-se no verbo). As consideraçóes que fizemos acima sobre perguntas com as palavras interrogativas podem ser estendidas, então, às perguntas sim / não. As respostas tanto de $\mathrm{B}$ quanto de B' tem como valor de foco o valor ordinário da pergunta de A, havendo assim congruência.

Considere agora o seguinte exemplo:

(26) A: Onde estão os meninos?

B: O JONAS (es)tá em CASA.

Nesse caso, B responde apenas parcialmente a pergunta de A, já que informa sobre a localização de apenas um dos meninos. De tal resposta, é natural inferimos que $\mathrm{B}$ desconhece (ou não quer informar) o local onde estão os demais meninos. Note que na resposta de B, Jonas recebeu um acento especial (que 
representaremos por letras maiúsculas sublinhadas), sendo pronunciado com uma entonação peculiar. ${ }^{6}$ A esse acento, Büring (1997) chama de acento de tópico (contrastivo / parcial) e uma resposta como a de B em (26) é representada da seguinte forma: ${ }^{7}$

$[\mathrm{O} \text { Jonas }]_{\mathrm{T}}$ está em $[\mathrm{casa}]_{\mathrm{F}}$

Antes de explicitarmos em que condiçôes tal acento pode ser usado, vamos a mais um exemplo, adaptado do autor:

(28) A: (Você acha que) o Jonas usaria esse terno?

B: (Bem), EU NÃO usaria.

Novamente, a resposta de $\mathrm{B}$ não é uma resposta direta à pergunta de $\mathrm{A}$ e, não fosse pelo acento de tópico em $e u$, tal resposta seria inadequada. Entretanto, com o acento (chamado frequentemente de tópico contrastivo), o falante parece veicular algo como o seguinte: o Jonas, eu não sei, mas...

Para capturar essas intuições e entender as condiçōes de uso desse acento de tópico, Buring propóe um terceiro valor semântico, chamado de valor de tópico, [[ ]]t. No caso de sentenças declarativas, o valor de tópico é um conjunto cujos membros são conjuntos de proposições. Em termos informais, cada um desses conjuntos pode ser obtido a partir das sentenças que entram na computação do valor de foco, substituindo o item que recebe o acento de tópico por alternativas do mesmo tipo. Por exemplo, no caso de (28), os membros do valor de tópico seriam da forma $\{\mathrm{X}$ usaria, $\mathrm{X}$ não usaria\}, com $\mathrm{X}$ sendo substituído por nomes de indivíduos. Assumindo aqui que as únicas alternativas relevantes nesse caso sejam eu e Jonas, obtemos o seguinte:

(29) $\left[\left[[\mathrm{eu}]_{\mathrm{T}}[\text { não }]_{\mathrm{F}} \text { usaria }\right]\right]^{\mathrm{t}}=$

$\{\{$ eu usaria, eu não usaria $\},\{$ Jonas usaria, Jonas não usaria $\}\}$

Note que os elementos do conjunto acima correspondem aos valores ordinários de duas perguntas, a saber, você usaria esse terno? e O Jonas usaria esse terno?. Note ainda que a primeira dessas perguntas foi respondida (negativamente) por $\mathrm{B}$, enquanto que a segunda, que corresponde à pergunta feita por A, foi deixada em aberto. Conforme notamos acima, esse deixar em aberto ou trazer uma pergunta à tona é o que caracteriza o uso do acento de tópico. Büring formaliza essa intuição por meio da seguinte condição: ${ }^{8}$ 
(30) Dada uma sentença $S$ contendo um tópico contrastivo, deve haver pelo menos um elemento em aberto em [[ $S$ ] ] $]^{\mathrm{t}}$ após o enunciado de $S$.

Como se pode notar, essa condição foi observada no caso de (28). O mesmo se pode dizer de (26). Para ver o porquê disso e a fim de evitar complexidades desnecessárias, imagine que os meninos em questão sejam Jonas, Pedro e Lucas e que os locais em que eles possam estar sejam em casa ou na rua. Assim teremos:

$\left[\left[\mathrm{O} \text { [Jonas }_{\mathrm{T}} \text { está em }[\text { casa }]_{\mathrm{F}}\right]\right]^{\mathrm{t}}=$

$\{\{$ Jonas está em casa, Jonas está na rua $\}$,

\{ Pedro está em casa, Pedro está na rua \},

\{Lucas está em casa, Lucas está na rua \}\}

Dada a pergunta que A fez em (26), é natural supor que o paradeiro dos meninos é uma questão em aberto. B responde apenas parcialmente a essa questão, informando sobre o paradeiro de Jonas. Com isso, permanecem abertas as questōes onde está Pedro? e onde está Lucas?. Como se pode ver em (31), ambas as questões são elementos pertencentes ao valor de tópico da resposta de $\mathrm{B}$, o que a torna adequada, em conformidade com a condição em (30).

\section{Expressões Lexicalmente Contrastivas}

De posse desse aparato teórico, voltemos aos exemplos relevantes da primeira seção.

(32) A: Dá pra ver que seu menino é filho único.

B': Não! Eu tenho mais de um filho.

B": \# Não! Eu tenho pelo menos dois filhos.

Após negar a afirmação de A, tanto B' quanto B” confirmam sua posição, já que alguém dizer que tem mais de um / pelo menos dois filhos é o mesmo que alguém negar que tenha um único filho (pressupondo, claro, a existência de um filho). Por que então a resposta de B" soa estranha, dando a impressão de que este desconhece o número exato de filhos que tem? Minha proposta é a seguinte: apesar de as sentenças usadas por B' e B' terem o mesmo valor semântico ordinário, seus valores de tópico são distintos. A razão para isso é que o 
modificador no mínimo é lexicalmente marcado com um acento de tópico, o que tem repercussões na interpretação de enunciados de que participa.

Conforme discutido na seção anterior, ao usar um acento de tópico, o falante propõe (implicitamente) uma questão a ser debatida. O que mostraremos a seguir é que, no caso de (32), a questão colocada em pauta não condiz com o que se espera ser de conhecimento do falante, causando, assim, um efeito de estranhamento. Passemos aos detalhes.

Já vimos que itens marcados com acentos de tópico ou foco introduzem alternativas. Nomes próprios, por exemplo, geram como alternativas outros nomes próprios, e operadores assertivos, como os de afirmação e negação, são alternativas um do outro. No caso de no mínimo, proponho que a alternativa em questão seja a expressão que funciona como seu oposto: no máximo. A intuição por traz disso é a seguinte: dizer que a cardinalidade de um certo conjunto é $n$ é o mesmo que dizer que essa cardinalidade é no mínimo $n$ e no máximo $n$. Marcar uma expressão como no minimo com um acento de tópico é torná-la uma expressão inerentemente contrastiva, caracterizando parcialmente a cardinalidade de um conjunto, impondo um valor mínimo, e levantando a questão sobre seu valor máximo. Note o paralelo com o exemplo (26), discutido mais acima. Naquele exemplo, a pergunta era sobre o paradeiro do grupo de meninos formado por Jonas, Pedro e Lucas, enquanto a resposta era apenas sobre Jonas, tendo seu nome recebido um acento de tópico, o que levantou a questão sobre o paradeiro de Pedro e Lucas.

Com isso em mente, vejamos as consequências da proposta acima. Iremos considerar duas possibilidades de análise para a sentença em (33):

(33) Eu tenho no mínimo dois filhos.

Na primeira, o foco recai sobre o operador assertivo, como representado abaixo:

(34) Eu [tenho $]_{\mathrm{F}}[\text { no mínimo }]_{\mathrm{T}}$ dois filhos.

Nesse caso, o valor de tópico seria o seguinte:

(35) $[[(34)]]^{t}=$

\{\{ eu tenho no mínimo dois, eu não tenho no mínimo dois $\}$,

\{ eu tenho no máximo dois, eu não tenho no máximo dois \}\} 
Note agora que o segundo elemento do conjunto acima corresponde à seguinte pergunta: o número de filhos do falante é ou não é no máximo dois (ou seja, menor que três)? Como já discutimos na seção anterior, um acento de tópico só é licenciado se houver uma questão deixada em aberto no valor de tópico da sentença. Como a pergunta correspondente ao primeiro conjunto em (35) foi respondida de forma afirmativa pelo falante, somos, então, levados à conclusão de que o falante pergunta (implicitamente) se seus filhos são dois ou mais de dois. Disso, podemos inferir que o falante ignora o número exato de filhos que tem.

É por essa ser uma situação inusitada, que a resposta causa estranhamento. Vamos agora considerar a possibilidade de que o foco recaia sobre o numeral:

(36) Eu tenho $[\text { no mínimo }]_{\mathrm{T}}[\text { dois }]_{\mathrm{F}}$ filhos.

Para o valor de tópico, teríamos o seguinte:

(37) $[[(36)]]^{\mathrm{t}}=$

$\{\{$ no mínimo 2, no mínimo 3 , no mínimo $4, \ldots\}$,

$\{$ no máximo 2 , no máximo 3 , no máximo $4, \ldots\}\}$

Nesse caso também o uso de no mínimo implica ignorância do falante sobre o número de filhos que ele tem. Ele afirma que tal número é maior ou igual a dois, e pergunta se esse número é menor ou igual a dois, menor ou igual a três, menor ou igual a quatro... Em outras palavras, o falante garante que tem dois filhos e deixa em aberto a possibilidade de esse número ser maior.

O uso de no máximo é regulado de maneira análoga, sua alternativa sendo a expressão no mínimo.

(38) Eu $[\text { tenho }]_{\mathrm{F}}[\text { no máximo }]_{\mathrm{T}}$ dois filhos.

(39) $[[(38)]]^{\mathrm{t}}=$

\{\{ eu tenho no máximo dois, eu não tenho no máximo dois \},

\{ eu tenho no mínimo dois, eu não tenho no mínimo dois \}\}

(40) Eu tenho [no máximo $]_{\mathrm{T}}[\mathrm{dois}]_{\mathrm{F}}$ filhos. 
(41) $[[(40)]]^{\mathrm{t}}=$

$\{\{$ no máximo 2, no máximo 3 , no máximo $4, \ldots\}$,

$\{$ no mínimo 2, no mínimo 3, no mínimo $4, \ldots\}\}$

Nesses casos, o falante garante que tem dois filhos e deixa em aberto a possibilidade de que esse número seja menor que dois.

\section{Conclusão}

Valendo-nos de princípios gerais que regulam o uso de perguntas e respostas, mostramos neste artigo como capturar uma assimetria inesperada entre o uso de modificadores cardinais como no mínimo e no máximo, por um lado, e mais de e menos de, por outro. A ideia central apresentada aqui foi a de que no mínimo e no máximo trazem do léxico um acento de tópico, o que faz com que seus usos estejam sujeitos a condiçóes que o uso de mais de e menos de não estão. Dessa forma, a despeito de contribuírem de maneira semelhante para o conteúdo proposicional das sentenças de que fazem parte, seus valores pragmáticos são distintos. Em particular, mostramos como o uso de no mínimo e no máximo legitimam sempre uma inferência de que o falante não está de posse de uma determinada informação. Quando, por razões extralinguísticas, essa inferência se torna implausível, surge um efeito de estranhamento, o qual ilustramos e discutimos neste artigo.?

\section{Notas}

${ }^{1}$ Sobre a origem, semântica ou pragmática, da exatidão associada ao uso de sintagmas cardinais não modifiados como três carros, ver Horn (1972), Horn (1989), Horn (1992), Levinson (2000), Geurts (2006), Ferreira (2008), para discussão e apresentação de pontos de vistas distintos.

${ }^{2}$ Ainda que tal sugestão pareça mais forte com o uso de no mínimo / no máximo.

${ }^{3}$ Em Geurts e Nouwen (2007), as contrapartes em inglês de no mínimo / no máximo at least / at most são tratadas como expressões modais, e uma sentença como John owns at least 3 cars tem por sentido literal algo como o seguinte: é necessário que o João tenha três ou mais carros e é possível que ele tenha mais de três carros. O fato de que esse caráter modal desaparece em certos ambientes parece ser um problema para a análise dos autores, como eles mesmos reconhecem em suas consideraçōes finais. 
${ }^{4}$ Vou utilizar letras em tipos normais para representar sentenças e em itálico para representar seus conteúdos proposicionais.

${ }^{5} \mathrm{Na}$ prática essas respostas são substituídas pelo simples uso do verbo acompanhado ou não da negação, como em chegou, não chegou, ou mesmo pelo uso das formas sim e não desacompanhadas.

${ }^{6}$ Não cabe aqui caracterizar foneticamente tal acento. Confiarei na intuição do leitor de que Jonas, na resposta de $\mathrm{B}$ em (26), não é pronunciado da mesma forma em que é pronunciado na resposta de $\mathrm{B}$ no diálogo a seguir:

(i) A: Onde está o Jonas?

B: O Jonas está em casa.

${ }^{7}$ Não se deve confundir essa noção de tópico contrastivo / parcial com uma outra noção de tópico discursivo (que não estamos discutindo neste texto) em que o elemento topicalizado aparece no início da sentença e retoma um indivíduo ou objeto saliente no contexto, como no diálogo abaixo:

(i) A: E o Jonas, por onde ele anda?

B: O Jonas, eu acho que se mudou do Brasil.

${ }^{8}$ Buring (2003) reformula essa condição, mas para os nossos propósitos ela é suficiente.

${ }^{9}$ Deixo como tópico para pesquisa futura o estudo do comportamento de no mínimo / no máximo em estruturas mais complexas, por exemplo, a restrição e o escopo de quantificadores (ver exemplo (14) da seção 2), incluindo os casos analisados por Geurts e Nouwen (2007) envolvendo concordância modal.

\section{Referências}

BURING, D. The meaning of topic and focus. London: Routledge, 1997.

BURING, D. On D-Trees, beans, and B-accents. Linguistics \& Philosophyn, v. 26, p. 511-545, 2003.

FERREIRA, M. Sintagmas cardinais complexos e implicaturas escalares. 2008. Disertação (Mestrado) - Universidade de São Paulo, 2008.

GEURTS, B. Take five: the meaning and use of a number word. In: VOGELLER, S.; TASMOWSKI, L. (Ed.). Non-definiteness and plurality. Amsterdam/Philadelphia: Benjamins, 2006. 
GEURTS, B.; NOUWEN, R. At least et al.: The semantics of scalar modifiers. Language, n 83, p. 533-559, 2007.

HAMBLIN, C. L. Questions in Montague Grammar. Foundations of Language, n. 10, p. 41-53, 1973.

HORN, L. The said and the unsaid. In: BARKER, C.; DOWTY, D. (Ed.). SALT II, 1992.

HORN, L. A Natural History of Negation. Chicago. IL: University of Chicago Press, 1989.

HORN, L. On the Semantic Properties of Logic operators in English. 1972. Tese (Doutorado) - UCLA, 1972.

LAKA, I. Negation in Syntax. 1990. Tese (Doutorado) - MIT, 1990.

LEVINSON, S. Presumptive Meanings. Cambridge, MA: MIT Press, 2000.

MARTINS, A. M. Enclisis, VP-deletion and the nature of Sigma. Probus, n. 6, p. 173-205, 1994.

NILSEN, Oystein. At least: free choice and lowest utility. In: Proceedings of the European Summer School in Logic, Language, and Information Workshop on Quantifier Modification, Dublin, 2007.

ROOTH, M. Focus. In. LAPPIN, S. (Ed.). Handbook of Contemporary Semantic Theory, Oxford: Blackwell, 1996.

ROOTH, M. Association with Focus. 1985. Tese (Doutorado) - University of Massachusetts at Amherst, 1985. 